\title{
Motor units in incomplete spinal cord injury: electrical activity, contractile properties and the effects of biofeedback
}

\author{
R B Stein, B S Brucker, D R Ayyar
}

\begin{abstract}
The electrical and contractile properties of hand muscles in a selected population of quadriplegic subjects were studied intensively before and after EMG biofeedback. Spontaneously active motor units and units that could only be slowly and weakly activated were observed in these subjects, in addition to units that were voluntarily activated normally. This suggests a considerable overlap of surviving motor neurons to a single muscle that are below, near or above the level of a lesion. Despite the common occurrence of polyphasic potentials and other signs of neuromuscular reinnervation, the average twitch tension of single motor units in hand muscles of quadriplegic subjects was not significantly different from that in control subjects. Nor did it increase after biofeedback training that typically increased the peak surface EMG by a factor of 2-5 times. The percentage of spontaneously active units was also constant. The surface EMG may be increased during biofeedback by using higher firing rates in motor units that can already be activated, rather than by recruiting previously unavailable motor units.
\end{abstract}

Recording from single motor units gives a unique window into the integration of inputs to human motor neurons in a variety of states. ${ }^{1-3}$ Although there have been a few studies of motor units following spinal cord injury, ${ }^{4-6}$ motor unit recordings in chronic, spinal cord injured patients (a year or more after injury) is rarely done clinically, perhaps because of the widely held view that the situation has stabilised and little prospect for improved function remains. However, treatment using biofeedback, based on surface EMG recordings from major muscle groups, combined with physiotherapy and electrical stimulation may improve function substantially in some patients. ${ }^{7-9}$ The efficacy of such treatment remains controversial and the neuronal mechanisms underlying any increase in the surface EMG signals through biofeedback are obscure.

The purpose of this study was to examine the electrical and contractile properties of single motor units in detail, using techniques developed over the last fifteen years. ${ }^{310}$ For this study subjects were selected who had incomplete spinal cord injuries and showed some activity in hand muscles, although hand function remained grossly defective more than a year after injury. The subjects selected had responded well to therapy including biofeedback of the larger muscle groups of the arm, but had not received any biofeedback to the hand muscles. They were studied before and after intensive biofeedback therapy to assess the nature of the functional deficits and possible neuronal mechanisms underlying any improvements shown.

\section{Methods}

Three hundred motor units were studied from 18 muscles of six adult, quadriplegic subjects (three male and three female; time since injury one to seven years; level of injury C4-C6). The subjects gave their written informed consent to participate in the study. The needles used for the quadriplegic subjects and the normal controls consisted of two teflon-coated stainless steel wires (diameter $=75 \mu \mathrm{m}$ ) inserted into a 25 gauge needle. The wires were fixed in place with epoxy, and ground flush with the barrel of the needle. Needles were gas sterilised before use. This design has proved to be a good compromise between the selectivity needed for single unit recording at medium to high force levels and the stability required to hold a unit for several minutes of voluntary contraction during spike-triggered averaging. ${ }^{10}$ Recordings were made bilaterally from thenar and first dorsal interosseous (FDI) muscles (about 10 units from each muscle studied) in which there was evidence of some voluntary electrical activity from surface EMG recordings.

Surface electrodes (Beckman type $\mathrm{Ag} / \mathrm{AgCl}$, $8 \mathrm{~mm}$ recording diameter) were placed over the muscles of interest on either side of the site where the needle was inserted. Recordings from these electrodes were used to check for synchronisation between motor units (see below) and to estimate the contribution of the single unit recorded by the needle to the surface EMG. Isometric force was recorded by means of a Grass FT03 strain gauge. The methods for recording force from the FDI muscle and the thenar muscles have been described previously..$^{111}$

The action potentials from a single motor unit were amplified (Leaf Electronics Model QT5B, Edmonton, Canada), highpass filtered using a $100 \mathrm{~Hz}$ RC filter and then discriminated using a Bak Electronics (Rockville, MD) Model DIS-1 Window Discriminator. Pulses from the discriminator were used to trigger 
signal averaging using a Cadwell Laboratories (Kennewick WA) Model 7400.

Four signals were averaged as shown in fig 1: 1) the motor unit action potential after a time delay of 5-10 ms (Bak Electronics Model AD-6 Analog Delay); 2) the surface recordings after a $100 \mathrm{~Hz}$ high-pass filter and time delay of $5-10 \mathrm{~ms} ; 3$ ) the same surface signals after additonally being full-wave rectified and 4) the force signals after being band-pass filtered over a range of $0 \cdot 16-100 \mathrm{~Hz}$. The averaging of the surface signals, both rectified and unrectified, permitted a test of motor unit synchronisation to be used. ${ }^{10}$ The use of AC filtering of the force records with a long time constant (1s) permitted the force fluctuations associated with the unit action potentials to be determined. If synchronisation is absent, this permits the motor unit twitch tension to be estimated. ${ }^{10}$

Biofeedback EMG measurements were obtained with gel-filled Beckman electrodes similar to those described above. Two active electrodes were placed over the belly of the muscle of interest (thenar or FDI) and a third ground electrode was placed on the distal end of the forearm. A prototype NeuroEducator manufactured by Therapeutic Technologies (Dayton, Ohio) was used to analyse and provide feedback of the EMG signal. The NeuroEducator measures the root mean square (RMS) voltage with an internal noise level of less than $0.2 \mu \mathrm{V}$ with a bandwidth of $10-10,000 \mathrm{~Hz}$, common mode rejection better than $140 \mathrm{~dB}$. EMG signals were integrated over $0 \cdot 1 \mathrm{~s}$, calibrated in $\mathrm{mV}$ and displayed on a colour monitor in the form of a continuous

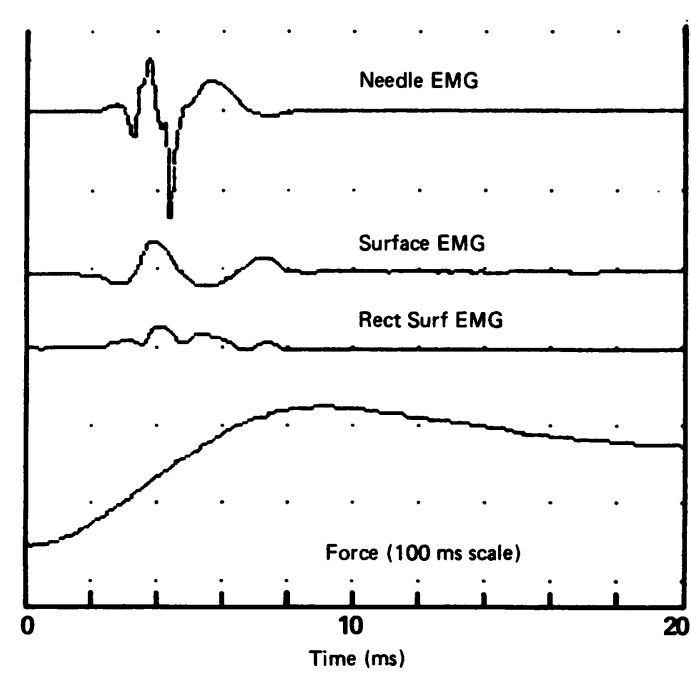

Figure 1 Action potentials from a single motor unit recorded with a needle electrode (top trace) were used to trigger an averaging program which averaged the needle EMG, the surface EMG before (trace 2) and after (trace 3) rectification and the force fluctuations in the muscle (trace 4). The top three traces were delayed $5 \mathrm{~ms}$. The bottom trace is on a slower time scale and provides a measure of the twitch tension of the motor unit, assuming that the discharges of different motor units are independent (no sychronisation). The peak-to-peak amplitudes are 1.38 vV (needle EMG) and $0.10 \mathrm{mV}$ (surface EMG) and the twitch tension is $66 \mathrm{mN}$. The absence of synchronisation can be checked using the surface EMG, as described in the text. Records from the FDI muscle of a quadriplegic subject (JB). line updated every $0 \cdot 1 \mathrm{~s}$ for a sweep of $20 \mathrm{~s}$ duration.

Subjects sat 1-2 $\mathrm{m}$ in front of the monitor and were instructed that the line on the screen represented the amount of signal recorded from the muscle being studied. They were to make the line go as high as possible when told to do so and to stop when told. After the first try a cursor was placed on the monitor at the highest recorded level of EMG and verbal reinforcement was given for EMG responses that exceeded the maximum level, after which the cursor was set at the new maximum. Training sessions lasted 45 minutes to an hour, during which 5-10 training trials for the thenar and FDI were conducted. Each trial consisted of approximately a 10s attempt to increase the EMG signal, preceded and followed by a five second rest. Inter-trial intervals were 2-4 minutes. Data were stored on disc and a hard copy of average EMG responses every $0.5 \mathrm{~s}$ was produced for further data analysis.

\section{Results}

As the patients who were selected for detailed study had continuing difficulty with hand function, it was not surprising that the number of motor units encountered at any needle location was grossly reduced and not uniform. Activity could be absent in large regions of one muscle, for example, first dorsal interosseous (FDI), but present in an adjacent muscle, adductor policis, innervated by the same nerve from approximately the same spinal roots. The number of active motor units could also vary enormously between diffeent portions of the same muscle (for example, deep and superficial compartments).

Active units could be divided into three classes: 1) those under approximately normal control with a clear threshold for activation and a range of firing rates comparable to those in control subjects; 2) spontaneously active units that could not be activated by the subject. These tended to fire at low rates $(<5$ impulses/s), but the rate could often be increased by muscle stretch and/or by spasms which involved the hand muscles. Overall, $20 \%$ of the units studied in these quadriplegic subjects were spontaneously active, but the numbers varied from $0-50 \%$ in different muscles and different subjects; 3 ) units that could only be slowly and weakly activated. When a subject was asked to turn off a unit, it might take 5-10s for the unit to stop firing. These units comprised less than $5 \%$ of the units overall, but could represent up to $20 \%$ of the units in some muscles.

A similar, long latency was often required to reactivate the unit, and unusual methods might be used. In an exteme example, a subject noticed that when she tried to move her big toe, her thumb also moved and this proved the best way for her to activate some units in her thenar muscles. When activated, these thenar units tended to fire at low initial rates similar to those of the sponateously active units, but with effort the rate might 
increase to more normal values. Thus it was difficult to determine with any assurance the percentage of motor units which fell into this category, but again it varied greatly between muscles and subjects.

Once a unit was activated, by whatever means, the motor unit action potential recorded by the needle electrode (trace 1 in fig 1) was used to trigger signal averaging of the unrectified surface EMG (trace 2), the rectified surface EMG (trace 3 ) and the force fluctuations in the muscle (trace 4). The reason for averaging the surface EMG both before and after rectification was to test for the presence of motor unit synchronisation.

Absence of motor unit synchronisation The rectified surface EMG may contain 1) a nonzero steady level corresponding to the mean rectified EMG at the surface of the muscle, 2) one or more peaks corresponding to the phases of the unrectified surface EMG and 3) a broad peak representing the tendency of motor units in the muscle to fire at about the same time (synchronisation). In the recordings from this and other motor units of the quadriplegic subjects, no broad peak (item 3 above) was seen, so evidence of synchronisation was lacking.

Assuming that the motor units fired independently of each other, the force change seen in trace 4 gives an estimate of the twitch force and contraction time of the single motor unit shown above. Subjects were required to maintain firing rates less than 8-10 impulses/s to minimise the effects of partial fusion on estimated twitch tensions. ${ }^{12}$ Trace 4 has a time scale that is five times longer than the other traces and no delay was used, so the action potentials were largely over before force generation began.

Polyphasic potentials In trace 1 of fig 1 the average motor unit action potential was highly polyphasic (six phases separated by zero crossings can be seen). This was quite common as shown in fig 2 where averaged motor unit action potentials for three successive units recorded from the same subjects are displayed. Motor unit action potentials from the first two impulses are highly polyphasic, whereas the third had a relatively simple triphasic shape. However, this unit had a small linked potential occurring about $5 \mathrm{~ms}$ after the main action potential. Such linked potentials were added to the number of phases if they represented additional zero crossings and were significantly above the noise level.

On average, the motor unit action potentials

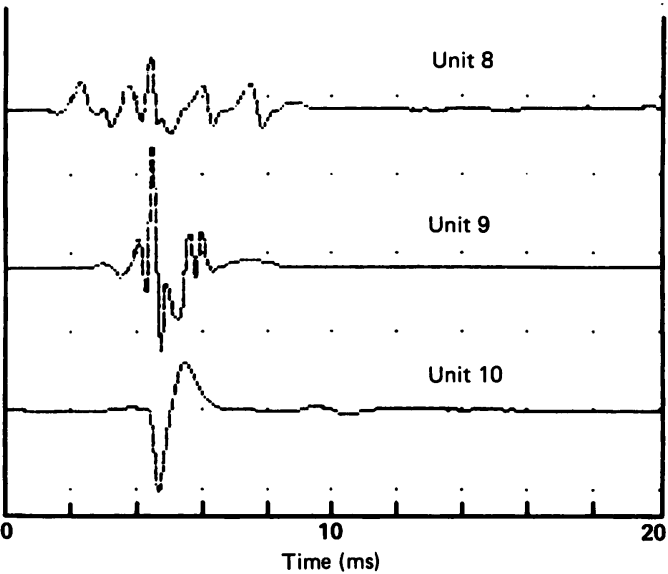

Figure 2 Averaged motor unit action potentials recorded in succession from a localised region of the thenar muscles of the same quadriplegic subject as in fig 1. The first two are highly polyphasic, having 12 and 11 phases respectively separated by clear crossings of the baseline. The third unit had only two prominent phases although a small linked potential can be observed, delayed by $5 \mathrm{~ms}$ from the main potential. The peak to peak amplitudes of the potentials are 0.10 (unit 8), 0.53 (unit 9) and 0.17 mV (unit 10).

recorded from 16 muscles of subjects with spinal cord injuries had a mean and standard deviation (SD) of $5.5(1 \cdot 1)$ phases (the mean values ranged for different muscles from 4.4 to 9.6). In contrast, the values with the same electrodes had a mean of $4.1(0.1)$ in control subjects and a range from 3.9 to $4 \cdot 3$. Thus there was no overlap in the two samples, but the values in the quadriplegic subjects varied from nearly normal to grossly abnormal.

Polyphasic potentials are a sign that the impulses from muscle fibres belonging to the motor unit recorded by the needle occur at slightly different times. This can arise, for example, from the presence of fine and hence slowly conducting branches of nerve fibres that sprout to innervate nearby, denervated muscle fibres.

Force generation If substantial sprouting at nerve terminals had occurred, the force generated by motor units should increase considerably. Table 1 shows the values found for subjects in which spike-triggered averaging was used to estimate motor unit twitch tension from hand muscles, as explained in the Method and fig 1 . The values of motor unit force for the quadriplegic subjects were not significantly different from those of normal subjects.

The force of the spontaneously active units described above for quadriplegic subjects was slightly smaller than the average unit in both

Table 1 Mean twitch tensions of motor units from FDI and thenar muscles in normal subjects and quadriplegic subjects before and after receiving EMG biofeedback training

\begin{tabular}{llllr}
\hline & Number of subjects & Units/subject & Mean Force (SE) (mN) Range (mN) \\
\hline FDI Muscles & & & & \\
Normal subjects & 7 & $5-72$ & $28(7)$ & $13-64$ \\
Quads before biofeedback & 8 & $5-12$ & $23(6)$ & $8-57$ \\
Quads after biofeedback & 6 & $5-13$ & & $11-32$ \\
Thenar Muscles & 5 & $14-30$ & $16(5)$ & $6-34$ \\
Normal subjects & 5 & $7-13$ & $12(2)$ & $7-19$ \\
Quads before biofeedback & 6 & $7-13$ & $15(4)$ & $6-26$ \\
Quads after biofeedback & 6 & &
\end{tabular}




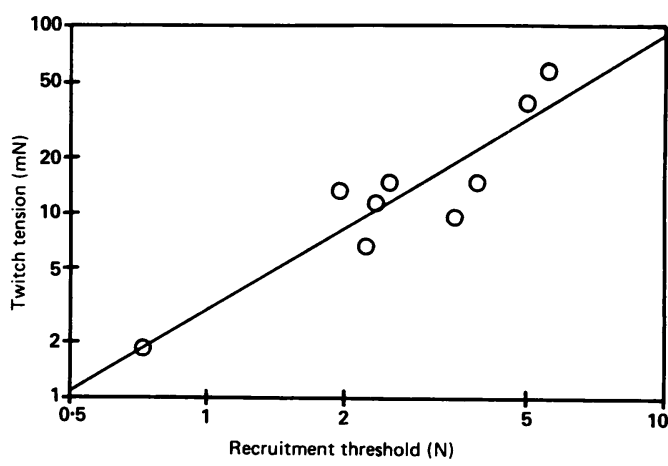

Figure 3 Motor units in quadriplegic subjects were recruited in an orderly fashion according to the size of their twitch tension, estimated by spike-triggered averaging. Recruitment threshold is the voluntary force level (N) at which a motor unit began to fire regularly. Both axes are logarithmic. Subject TDM, left thenar muscles.

the force generated $(86 \%)$ and the surface EMG recorded $(84 \%)$. These differences from the average values were significant $(p<0.05$ using Student's $t$-test). Possible explanations for these findings will be considered in the Discussion.

The order of recruitment was also normal in that small units were recruited first (their thresholds for recruitment were low) and increasingly large units were recruited at progressively large threshold force values (fig 3). An orderly pattern of recruitment is seen in patients after pressure block of the nerve or when the number of motor units is reduced in amyotrophic lateral sclerosis. ${ }^{13}$ An apparently random pattern of recruitment is seen after cutting and resuturing a nerve, but this may be due to misdirection of regenerating nerve fibres. ${ }^{14}$

Biofeedback The patients, whose hand muscles were studied in detail, were given intensive biofeedback using surface EMG and computerised displays of the RMS voltages recorded during periods of voluntary activity lasting about 10s (see Method). Figure 4 shows the range of peak EMG values (averaged over three successive $0.5 \mathrm{~s}$ intervals) recorded on eight sessions over a three week period with one

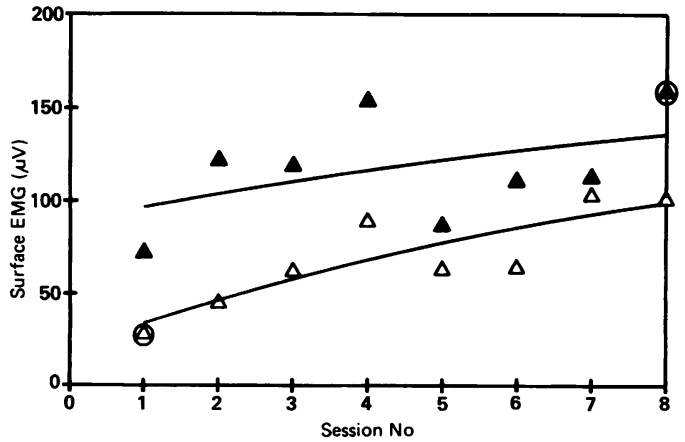

Figure 4 Peak surface EMG averaged over a 1.5s interval during biofeedback sessions over a three week period. The largest (filled triangles) and smallest (open triangles) peak values recorded in trials lasting 20 s are plotted for each session and the extreme values recorded overall are circled. The best fitting parabola in the least mean squares sense is plotted for both data sets. Subject $J B$, left FDI muscle. subject. The circled values show a more than five-fold increase from a low, initial value of 29 $\mu \mathrm{V}$ to a high, final value of $160 \mu \mathrm{V}$. The curves are parabolas fitted so as to minimise the root mean square error to the maximum and minimum values for each session. They are included more to guide the eye than to suggest a particular form for the increase, but they are more representative of the trend than simply picking high and low values.

Figure 4 was typical of the 14 hand muscles in which biofeedback was attempted. The ratio of highest to lowest values for these muscles was 5.6(1.0) (mean SE) with a range from 1.4 to 13.5. A more conservative approach is to fit a curve, as was carried out in fig 4 , to all the stored values. When this was done the mean ratio on the final and initial sessions was 2.0 $(0 \cdot 3)$ (range from $1 \cdot 0$ to $4 \cdot 3$ ).

In contrast, when we examined the twitch force of the single motor units that the subjects could activate in a follow up session after the biofeedback, no significant difference was seen (table 1). Nor was the EMG contributed by these single units to the surface recording significantly higher. The mean values (SE) were 109 (36) $\mu \mathrm{V}$ before biofeedback and 106 (31) $\mu \mathrm{V}$ after biofeedback. Finally, the percentage of spontaneously active units in the 14 muscles was $17 \%$ before biofeedback and $18 \%$ following biofeedback. The reasons for these results will now be discussed.

\section{Discussion}

This is the first study to consider in detail the contractile as well as the electrical properties of motor units following spinal cord injury, and the changes in motor unit properties that take place following biofeedback using surface EMG. The results provide information concerning several important questions. For example, are there distinct classes of motor units that survive spinal cord injury? What is the nature of the very slow recovery which is often seen in these patients? What are the neural mechanisms underlying increases in the surface EMG during biofeedback? Answers to these questions will be proposed in separate sections below and experiments will be suggested for future tests of the proposals.

Classes of motor units after spinal cord injury A mixed picture emerges in studying motor units of muscles innervated from levels of the spinal cord close to the injury. Some motor units are spontaneously active and modified by reflexes but not voluntary commands. Their motor neurons presumably lie below the level of injury and the spontaneous activity reflects the loss of the normal descending inhibition. Their somewhat smaller size, as measured by their force output or their EMG, probably does not imply that they are a distinct type of motoneuron. The reductions in size could easily result from their continuous activity at low firing rates. This pattern is well known to convert muscle fibres to oxidative metabolism, with the result that they are fatigue-resistant, but generate less force and contract more slowly. ${ }^{15}$ 
Other motor units in the immediate vicinity of spontaneously active ones seemed to be activated quite normally and presumably had motor neurons situated above the level of the injury. The presence of a third class of very slowly activated units is intriguing. By using fast cortico-spinal connections normal subjects can activate motor units in a fraction of a second, yet the quadriplegic subjects showed substantial numbers of units that could only be activated or deactivated with latencies up to about 10s. Reduced firing rates of motor units have been seen in a variety of central disorders, ${ }^{16-18}$ but previous work has not emphasised increased latencies of this magnitude.

Perhaps some slow pathways involving for example reticulospinal or other pathways were not as affected by the spinal injury and may be substituted for faster pathways. Certainly, the rather gross synergies often observed were consistent with this explanation. Substitute pathways might also be greatly facilitated by the generalised learning paradigm embodied in the biofeedback procedure. Activation of descending pathways by magnetic stimulation ${ }^{19} 20$ over the motor cortex or the cerebellum while recording from motor units would be interesting to confirm which pathways were operating in these patients and what their latencies were.

Regeneration and/or sprouting The presence of the classic electrophysiological signs for the growth of motor axons to reinnervate denervated muscle, such as polyphasic potentials, implies that substantial remodelling of neuromuscular connections took place. Although measurements in this study were done with specially designed needle electrodes, routine EMG examination with conventional needle electrodes also shows substantial numbers of polyphasic potentials, as well as increased durations and amplitudes of motor unit potentials (DR Ayyar, unpublished data). If unaffected motor neurons had sprouted extra connections to denervated muscle fibres, motor units that generated much more force might have been expected, but such units were not observed.

We also noted, however, that the orderly recruitment of motor units was retained, so the largest motor neurons in these weakened muscles, that should be able to generate the largest forces, may not have been activated voluntarily. In fact, in preliminary results from a study on another group of quadriplegic patients, ${ }^{21}$ the problem of incomplete recruitment was overcome by use of microstimulation. ${ }^{22}$ Average tensions up to five times the normal values were then observed in patients with greatly reduced numbers of motor units as a result of the spinal cord injury. The most likely explanation therefore for the polyphasic potentials is sprouting of terminals from unaffected motor neurons, but we cannot rule out the possibility of some regeneration from the spinal cord. The slow rate of recovery often observed over months or even years seems very slow to be fully accounted for by sprouting. It would be interesting to follow the time course of changes with modern electrophysiological techniques to determine the nature and the time course of the mechanisms that are operating.

What is increased during biofeedback? As demonstrated here, patients with incomplete spinal cord injuries can substantially increase the amount of surface EMG generated in a muscle through the use of biofeedback. A priori, several possible neuronal mechanisms can be suggested for this increase: 1 ) increased firing rates in the population of motor units that were activated before biofeedback, 2) increased numbers of motor units recruited to fire, 3 ) increased synchronisation of motor unit firing so that less cancellation occurs in the surface EMG, 4) an improved safety factor in neuromuscular transmission so that muscle fibres continued to fire for longer periods of time or at higher rates of activation, 5) sprouting of motor nerve terminals to innervate additional muscle fibres. Any of these factors could potentially increase the magnitude of the surface EMG, but needle recordings and other more sophisticated approaches are needed to distinguish among them.

Possibilities 3-5 can reasonably well be discounted from the experiments reported here, as will be discussed in turn. By comparing the rectified and unrectified surface EMG (fig 1) no evidence of synchronisation was obtained. A degree of synchronisation has been observed in some subject groups with this technique, ${ }^{23}$ although it is not common in the normal population. Other techniques involving crosscorrelations between units recorded on separate needle electrodes have suggested a higher degree of synchronisation in the normal population, ${ }^{24}$ but the cross-correlation techniques are difficult to quantify ${ }^{25}$ Finally, the greatly reduced numbers of motor units in these subjects means that there will tend to be less cancellation in the surface EMG, so synchronisation would not be expected to contribute appreciably to the large increases in surface EMG shown for example in Fig 4.

If neuromuscular transmission was insecure (possibility 4 above) a myasthenic type of response might occur with decreasing potentials even at low rates of repetitive activation. To use spike-triggered averaging, subjects had to activate motor units continuously for 3-5 minutes. Under such circumstances a decrease in motor unit amplitude might be expected, but was not observed. There was considerable jitter between the various phases of the polyphasic potentials, as would be expected after reinnervation, ${ }^{26}$ but there was no systematic trend in the amplitude.

Substantial increases in the surface EMG were observed in a single session of biofeedback lasting no more than an hour, and with the intensive treatment used here, the training was completed within one to three weeks. Sprouting of axons (possibility 5 above) would not occur within a single session and would be unlikely to contribute substantially over a week or two. There was no evidence of greatly enlarged motor units, either in terms of electrical or contractile amplitude following biofeedback, which also argues against sprouting taking place during the period of interest. 
Since an orderly recruitment of motor units according to size could be demonstrated in these subjects (fig 3), any extra units recruited should have been very large ones. The lack of change in the size of the motor units, either in terms of increased electrical or contractile properties recorded after biofeedback, also argues against a substantial recruitment of previously unavailable units as a result of biofeedback. Finally, no change was observed in the percentage of motor units which were spontaneously active, so this group was not brought under voluntary control.

Thus by a process of elimination the evidence points towards the first possibility listed above and suggests that the increased biofeedback scores may result mainly from increased firing rate of motor units that could previously be activated. Technical differences, however, are such that other possibilities cannot be completely excluded. The peak EMG values recorded during biofeedback were only measured over a $1.5 \mathrm{~s}$ period. In contrast, spiketriggered averaging required a contraction to be maintained for several minutes, so quite different mechanisms could be operative. For example, a voluntarily triggered spasm might be generated for a brief period, but not maintained. Similarly, during biofeedback muscles may be restrained or even stretched during effort, which could reflexly facilitate neurons in a way not matched during the needle EMG examinations. It is also technically very difficult to measure firing rates of motor units during brief, maximum voluntary contractions, but this should be attempted in future studies to confirm directly that increased rate is the most important factor in generating the greater surface EMG resulting from biofeedback.

We thank Drs Barth A Green, Walter J Levy and other members of the Miami Project to Cure Paralysis without whose help this study would not have been possible. Dr Blair Calancie kindly constructed the electrodes used in this study. Drs Calancie, Jack Jhamandas and George Elleker provided valuable suggestions on the manuscript. Support was received in part from the Miami Project, the Veterans Administration and the Medical Research Council of Canada.

1 Buchthal F, Schmalbruch H. Motor units of mammalian muscle. Physiol Rev 1980;60:90-142.

2 Freund H-J. Motor unit and muscle activity in voluntary motor control. Physiol Rev 1983;63:387-436.
3 Stein RB. Novel uses of EMG to study normal and disordered motor control. Can J Neurol Sci 1988;15:95-8. 4 Brandstater ME, Dinsdale SM. Electrophysiological studies in assessment of spinal cord lesions. Arch Phys Med Rehabil 1976;57:70-74.

5 Hunter J, Ashby P. Secondary changes in segmental neurons below a spinal cord lesion in man. Arch Phys Med Rehabil 1984;65:702-5.

6 Taylor RG, Kewalramani LS, Fowler WM. Electromyographic findings in lower extremities of patients with high spinal cord injury. Arch Phys Med Rehabil 1974;55: 16-23.

7 Cybulski GR, Penn RD, Jaeger RJ. Lower extremity functional neuromuscular stimulation in cases of spinal cord injury. Neurosurgery 1984;15:132-46.

8 Glaser RM. Exercise and locomotion for the spinal cord injured. Exerc Sport Sci Rev 1985;13:263-303.

9 Goldsmith MF. Computerized biofeedback training aids in spinal injury rehabilitation. $J$ Amer Med Ass 1985;253: 1097-9.

10 Milner-Brown HS, Stein RB, Yemm R. The contractile properties of human motor units during voluntary
isometric contractions. $J$ Physiol (Lond) 1973;228: 285-306.

11 Thomas CK, Ross BH, Calancie B. Human motor-unit recruitment during isometric contractions and repeated recruitment during isometric contractions and repeated

12 Calancie B, Bawa P. Limitation of the spike triggered averaging technique for obtaining motor unit twitch averaging technique for obtaining
profiles. Muscle Nerve 1976;9:78-83.

13 Milner-Brown HS, Stein RB, Lee RG. Pattern of recruiting human motor units in neuropathies and motorneurone disease. J Neurol Neurosurg Psychiatry 1974;37:665-9.

14 Thomas CK, Stein RB, Gordon T, Lee RG, Elleker MG. Patterns of reinnervation and motor unit recruitment in human hand muscles after complete ulnar and median nerve section and resuture. J Neurol Neurosurg Psychiat 1987;50:259-68.

15 Vrbova G, Gordon T, Jones R. Nerve-Muscle Interaction. London: Chapman and Hall, 1978.

16 Dietz V, Hillesheimer W, Freund H-J. Correlation between tremor, voluntary contraction and firing pattern of motor units in Parkinson's disease. J Neurol Neurosurg motor units in Parkinson's
Psychiatry 1974;37:927-37.

17 Rosenfalck A, Andreassen S. Impaired regulation of force and firing pattern of single motor units in patients with spasticity. J Neurol Neurosurg Psychiatry 1980;43:907-16.

18 Petajan JH. Motor unit control in movement disorders. In: J $\mathrm{E}$ Desmedt, ed. Motor control mechanisms in health and disease. Advances in Neurology, Vol 39. New York: Raven Press, 1983:897-905.

19 Barker AT, Jalinous R, Freeston IL. Non-invasive magnetic stimulation of the human motor cortex. Lancet 1985; i: 1106-7.

20 Hess CW, Mills KR, Murray NMF. Responses in small hand muscles from magnetic stimulation of the human brain. $J$ Physiol 1987;388:397-419.

21 Yang JF, Stein RB, Jhamandas J. New methods to estimate the number of motor units in a human hand muscle. Soc the number of motor units in

22 Taylor A, Stephens JA. Study of human motor unit contractions by controlled intramuscular microstimulation. Brain Res 1976;117:331-5.

23 Milner-Brown HS, Stein RB, Lee RG. Synchronization of human motor units: possible roles of exercise and supraspinal reflexes. Electroencephal Clin Neurophysiol 1975;38:549-69.

24 Datta AK, Fleming JR, Hortobagyi T, Stephens JA. Shortterm synchronization of high-threshold motor units in human first dorsal interosseus muscle recorded during steady voluntary isometric contractions. J Physiol (Lond) 1985;366:22.

25 Davey NJ, Ellaway PH, Stein RB. Statistical limits for detecting change in the cumulative sum derivative of the detecting change in the cumulative sum derivative of the peristim.

26 Stalberg E, Trontelj JV. Single Fibre Electromyography. Woking, United Kingdom: Mirvalle Pres, 1979. 